\title{
Gluon matter distribution in the proton and pion from extended holographic light-front QCD
}

\author{
Guy F. de Téramond $\odot,{ }^{1}$ H. G. Dosch, ${ }^{2}$ Tianbo Liu $\odot,{ }^{3, *}$ Raza Sabbir Sufian $\odot,{ }^{4,5, \dagger}$ \\ Stanley J. Brodsky $\odot,{ }^{6}$ and Alexandre Deur ${ }^{5}$
}

(HLFHS Collaboration)

${ }^{1}$ Laboratorio de Física Teórica y Computacional, Universidad de Costa Rica, 11501 San José, Costa Rica
${ }^{2}$ Institut für Theoretische Physik der Universität, D-69120 Heidelberg, Germany
${ }^{3}$ Key Laboratory of Particle Physics and Particle Irradiation (MOE), Institute of Frontier and
Interdisciplinary Science, Shandong University, Qingdao, Shandong 266237, China
${ }^{4}$ Department of Physics, William \& Mary, Williamsburg, Virginia 23187, USA
${ }^{5}$ Thomas Jefferson National Accelerator Facility, Newport News, Virginia 23606, USA
${ }^{6}$ SLAC National Accelerator Laboratory, Stanford University, Stanford, California 94309, USA

(Received 19 July 2021; accepted 8 November 2021; published 6 December 2021)

\begin{abstract}
The holographic light-front QCD framework provides a unified nonperturbative description of the hadron mass spectrum, form factors and quark distributions. In this article we extend holographic QCD in order to describe the gluonic distribution in both the proton and pion from the coupling of the metric fluctuations induced by the spin-two Pomeron with the energy momentum tensor in anti-de Sitter space, together with constraints imposed by the Veneziano model, without additional free parameters. The gluonic and quark distributions are shown to have significantly different effective QCD scales.
\end{abstract}

DOI: 10.1103/PhysRevD.104.114005

\section{INTRODUCTION}

The gluonic composition of hadrons plays a key role in understanding the confining phase of quantum chromodynamics (QCD), which is still an unresolved issue in modern particle physics. A key nonperturbative feature of color-confining hadron dynamics is the intrinsic gluon distribution which exists in hadrons over a time scale independent of the resolution of the external probe. The coupling of the rank-two energy-momentum tensor (EMT), the tensor which couples to gravity [1-3], provides fundamental constraints on the quark and gluon generalized parton distribution functions (GPDs) of a hadron [4-6]. Gravitational form factors (GFFs), the hadronic matrix elements of the EMT, describe the coupling of a hadron to the graviton and thus provide information on the dynamics of quarks and gluons within hadrons due to the internal shear forces and pressure distributions of the quarks and

\footnotetext{
*liutb@sdu.edu.cn

†sufian@jlab.org
}

Published by the American Physical Society under the terms of the Creative Commons Attribution 4.0 International license. Further distribution of this work must maintain attribution to the author(s) and the published article's title, journal citation, and DOI. Funded by SCOAP ${ }^{3}$. gluons [7-9]. In this article, we present an extended holographic light-front (LF) QCD framework for studying the gluon GFFs and provide predictions for the intrinsic gluon distributions of hadrons without introducing additional parameters.

In addition to the role of gluons as fundamental constituents and as the glue binding quarks into hadrons, the knowledge of gluon distributions within hadrons is also essential for the understanding of Higgs boson production [10] and particle production cross sections at smallmomentum fraction $x$. The near threshold production of heavy vector quarkonium [11], such as the $J / \psi$ and $\Upsilon$, is dominated by multiple gluon interaction identified with Pomeron exchange [12]: It is expected to shed light on the QCD trace anomaly at the origin of the proton mass [13]. On the other hand, the intrinsic gluon parton distribution function (PDF) of the pion, the lightest QCD bound state, is of particular theoretical interest for understanding nonperturbative aspects of QCD, such as the connection of the spontaneous breaking of chiral symmetry and confinement [14]. Because of the special role of the pion in QCD, there have been sustained efforts and proposals to explore its gluon distribution [15], along with that of the nucleon, one of the main goals of the upcoming Electron Ion Collider [16,17].

Holographic light-front QCD (HLFQCD), a nonperturbative framework based on the gauge/gravity 
correspondence [18] and its light-front holographic mapping [19-21], has the remarkable feature that it reproduces, within its expected precision, the hadronic spectra with the minimal number of parameters: the confining scale $\lambda$ and effective quark masses. The effective confining interaction for mesons and baryons is determined by an underlying superconformal algebraic structure [22-24], which leads to unexpected connections across the full hadron spectrum [25]. It reproduces the structure of hadronic spectra as predicted by dual models, most prominently the Veneziano model [26] with its typical features; linear Regge trajectories with a universal slope and the existence of "daughter trajectories".

The form factors (FFs) obtained within the HLFQCD framework can be expressed by Euler-Beta functions $[27,28]$, a feature also predicted by the generalized Veneziano model $[29,30]$, which includes the electromagnetic (EM) current and the FFs; $F_{\mathrm{EM}}(t) \sim B\left(\gamma, 1-\alpha_{\rho}(t)\right)$, where $\alpha_{\rho}(t)$ is the trajectory of the $\rho$-vector-meson, coupling to the quark current in the hadron. We emphasize that, in HLFQCD, the parameter $\gamma$, related to the falloff of the EM FF at large momentum transfer $t$, is not arbitrary, but fixed by the twist of the Fock state, $\gamma=\tau-1$, consistent with the exclusive counting rules [31,32]. The twist $\tau$ is the number of constituents $N, \tau=N(\tau=N+L$ for LF orbital angular momentum $L$ ).

The form of the quark distributions in the hadrons is heavily constrained by the analytic properties of the FFs. Furthermore, very natural assumptions, such as the incorporation of the inclusive-exclusive relation at large longitudinal momentum fraction $x$ [33], allowed us to predict, in a very satisfactory way, the quark distributions in mesons and nucleons [27,34], as well as the strange-antistrange and the charm-anticharm asymmetries $[35,36]$ in the nucleon. Notably, the preliminary NNPDF4.1 global analysis [37] indicates a valencelike intrinsic charm contribution $|u u d c \bar{c}\rangle$ in the nucleon, consistent with the intrinsic charm-anticharm asymmetry computed in [36]. In addition to higher twist, the sea quark distributions have distinctive Regge trajectories from the EM current couplings to the sea; $\alpha_{\phi}(t)$ and $\alpha_{J / \Psi}(t)$, respectively, for the strange and charm sea in the proton $[35,36]$. Likewise, the $|u u d g\rangle$ and $|u \bar{d} g\rangle$ Fock states should provide the leading contributions to the intrinsic gluon distributions of, respectively, the proton and pion coupled to a spin-two current with its characteristic Reggeized $t$-channel particle exchange.

In this article we extend our previous framework by incorporating gluonic matter with significantly different quarkonic and gluonic scales. Several models for nonperturbative QCD, see e.g., [38], predict that the static potential between quarks, $V_{\bar{q} q}$, or between gluons, $V_{g g}$, are substantially different, and scale with $N_{C}$ like the quadratic Casimir operator between the fundamental and adjoint representations, $\frac{V_{g g}}{V_{\bar{q} q}} \sim \frac{2 N_{C}^{2}}{N_{C}^{2}-1}$, a scaling confirmed by lattice calculation [39]. It is in qualitative accordance with the smaller slope of the Pomeron as compared to the Reggeons, $\alpha_{P} \ll \alpha_{\rho}$, therefore with different scales $\lambda_{q}$ and $\lambda_{g}$ for quarkonic and gluonic matter with $\lambda_{g} \gg \lambda_{q}$.

The rest of this paper is organized as follows. In Sec. II we study the perturbation of the anti-de Sitter (AdS) metric by an external spin-two current which couples to the EMT in the bulk. It allows us to identify the tensor Pomeron in the asymptotic physical boundary space with the induced metric fluctuations in AdS, and thus to determine the scale of the gluonic matter from the infrared deformation of the AdS metrics in terms of the Pomeron slope. By further imposing the structure of the generalized Veneziano amplitudes for a spin-two current, we are able to incorporate the Pomeron intercept in the analytic expression for the gravitational form factor $A^{g}\left(Q^{2}\right)$. Our results for the GFF for the proton and the pion are compared with recent lattice QCD computations. In Sec. III we extend the relation between FFs and GPDs [4-6] found in [27] to compute the gluon distribution functions. Our DGLAP evolved predictions for the proton and pion are then compared with global fits. Some final comments are included in Sec. IV.

\section{POMERON EXCHANGE AND GRAVITATIONAL FORM FACTORS}

As pointed out in [40], soft interactions play an important role in high-energy collisions. Especially, the trajectory for diffractive processes, the Pomeron, has a dominant role at small-angle high-energy scattering, which is beyond the applicability of perturbative QCD. Since the early days of QCD, Pomeron exchange was associated with two (or more) gluons [41-43]. The Pomeron couples as a rank-two tensor to hadrons [44-48] and couples strongly to gluons. It remains unclear whether there exists a relation between the soft [49] and hard [50-52] Pomerons and their crossover regime. It may be sufficient to consider only the soft Pomeron if one looks into the intrinsic gluon component of the nucleon structure functions, except, perhaps, for extremely small $x$ [50-54]. Therefore we use the soft Pomeron of Donnachie and Landshoff [49] with the effective Regge trajectory,

$$
\alpha_{P}(t)=\alpha_{P}(0)+\alpha_{P}^{\prime} t
$$

which is interpreted in QCD as a $J^{P C}=2^{++}$bound state of two gluons with intercept $\alpha_{P}(0) \simeq 1.08$ and slope $\alpha_{P}^{\prime} \simeq$ $0.25 \mathrm{GeV}^{-2}$ [40]. Pomeron exchange is identified as the graviton of the dual AdS theory [55-61] and the first hadronic state on the Pomeron trajectory is identified with the $2^{++}$glueball [62].

We start with the AdS gravity action with fivedimensional coordinates $x^{M}=\left(x^{\mu}, z\right)$ 


$$
S_{G}[g]=-\frac{1}{4} \int d^{5} x \sqrt{g} e^{\varphi_{g}(z)}(\mathcal{R}-\Lambda),
$$

modified by an exponential dilaton term $e^{\varphi_{g}(z)}$ in the string frame. The metric determinant is $g, \mathcal{R}$ is the scalar curvature and $\Lambda=-6 / R^{2}$, with $R$ the AdS radius. We consider the perturbation of the action (2) by an arbitrary external source at the AdS asymptotic boundary which propagates inside AdS space and couples to the EMT $[63,64]$. By performing a small deformation of the AdS metric about its AdS background, $g_{M N} \rightarrow g_{M N}+h_{M N}$, we obtain the effective action $S_{\text {eff }}[h, \Phi]=S_{g}[h]+S_{i}[h, \Phi]$

$$
\begin{aligned}
S_{g}[h]= & -\frac{1}{4} \int d^{5} x \sqrt{g} e^{\varphi_{g}(z)}\left(\partial_{L} h^{M N} \partial^{L} h_{M N}\right. \\
& \left.-\frac{1}{2} \partial_{L} h \partial^{L} h\right), \\
S_{i}[h, \Phi]= & \frac{1}{2} \int d^{5} x \sqrt{g} h_{M N} T^{M N}(\Phi),
\end{aligned}
$$

where $\Phi$ represents the matter field content. In deriving (3), we use the harmonic gauge $\partial_{L} h_{M}^{L}=\frac{1}{2} \partial_{M} h, h \equiv h_{L}^{L}$. The interaction term (4) describes the coupling of the EMT with the graviton probe in AdS - which we identify here with the Pomeron.

We consider the propagation of $h_{M N}$ with components along Minkowski coordinates and impose the additional gauge condition $\partial_{M} h=0$. From (3) we obtain the linearized Einstein equations

$$
-\frac{z^{3}}{e^{\varphi_{g}(z)}} \partial_{z}\left(\frac{e^{\varphi_{g}(z)}}{z^{3}} \partial_{z} h_{\mu}^{\nu}\right)+\partial_{\rho} \partial^{\rho} h_{\mu}^{\nu}=0,
$$

where $h_{\mu \nu}$ couples to the transverse and traceless part of the EMT in (4). The boundary limit of the graviton probe is a plane wave solution with polarization indices along the physical coordinates $h_{\mu}^{\nu}(x, z \rightarrow 0)=\epsilon_{\mu}^{\nu} e^{-i q \cdot x}$, $q^{2}=-Q^{2}<0$. Thus, $h_{\mu}^{\nu}(x, z)=\epsilon_{\mu}^{\nu} e^{-i q \cdot x} H\left(q^{2}, z\right)$, with $H\left(q^{2}=0, z\right)=H\left(q^{2}, z=0\right)=1$.

As an example, we represent the quark matter content of a pion by a scalar field $\Phi$ with action

$S_{q}[\Phi]=\int d^{5} x \sqrt{g} e^{\varphi_{q}(z)}\left(g^{M N} \partial_{M} \Phi^{*} \partial_{N} \Phi-\mu^{2} \Phi^{*} \Phi\right)$,

modified by the dilaton term $e^{\varphi_{q}(z)}$. It represents a pion with physical mass $P_{\mu} P^{\mu}=M_{\pi}^{2}$ and $\operatorname{AdS}$ mass $(\mu R)^{2}=-4$ [21]. The EMT follows from (6), with

$$
T_{M N}=\partial_{M} \Phi^{*} \partial_{N} \Phi+\partial_{N} \Phi^{*} \partial_{M} \Phi .
$$

It leads from the interaction term (4) to the transition amplitude

$$
\int d^{5} x \sqrt{g} h_{M N}\left(\partial^{M} \Phi_{P^{\prime}}^{*} \partial^{N} \Phi_{P}+\partial^{N} \Phi_{P^{\prime}}^{*} \partial^{M} \Phi_{P}\right) .
$$

The action $S_{q}[\Phi]$ (6) is modified by the dilaton term $e^{\varphi_{q}(z)}=e^{\lambda_{q} z^{2}}$ with $\lambda_{q}=1 / 4 \alpha_{\rho}^{\prime} \simeq(0.5 \mathrm{GeV})^{2}$, specific to the light front mapping to physical $(3+1)$-dimensional space in HLFQCD [21] and the constraints imposed by the superconformal algebraic structure [25]. On the other hand, the spin-two action $S_{g}[h]$ (3) describing the propagation of a gravitational fluctuation is only constrained by gauge invariance, therefore it is modified by a negative softwall dilaton profile [65] $e^{\varphi_{g}(z)}=e^{-\lambda_{g} z^{2}}$ with $\lambda_{g}=1 / 4 \alpha_{P}^{\prime} \simeq$ $1 \mathrm{GeV}^{2}$ from the Pomeron slope. The interaction term $S_{i}[h, \Phi](4)$ has no deformation term and does not introduce an additional scale. For a soft-wall profile $\varphi_{g}(z)=-\lambda_{g} z^{2}$ the solution to (5) is given by

$$
\begin{aligned}
H(a, \xi) & =\Gamma(2+a) U(a,-1, \xi) \\
& =a(2+a) \int_{0}^{1} d x x^{a-1}(1-x) e^{-\xi x(1-x),}
\end{aligned}
$$

where $a=Q^{2} / 4 \lambda_{g}, \xi=\lambda_{g} z^{2}$, and $U(a, b, z)$ is the Tricomi confluent hypergeometric function.

The usual expression of the GFF from the hadronic matrix elements of the EMT

$$
\left\langle P^{\prime}\left|T_{\mu}^{\nu}\right| P\right\rangle=\left(P^{\nu} P_{\mu}^{\prime}+P_{\mu} P^{\prime \nu}\right) A\left(Q^{2}\right),
$$

follows from extracting the delta function from momentum conservation in (8). We obtain for $A\left(Q^{2}\right)[63,64]$

$$
A_{\tau}\left(Q^{2}\right)=\int_{0}^{\infty} \frac{d z}{z^{3}} H\left(Q^{2}, z\right) \Phi_{\tau}^{2}(z)
$$

with $\Phi_{\tau}(z)$, the twist- $\tau$ hadron bound-state solution [21] and normalization $A_{\tau}(0)=1$.

To compute the gluon GFF, $A_{\tau}^{g}\left(Q^{2}\right)$, the Pomeron is assumed to couple mainly to the constituent gluon [44-48]. The lowest twist is the $\tau=4$ Fock state $|u u d g\rangle$ in the proton and the twist $\tau=3$ state $|u \bar{d} g\rangle$ in the pion, both containing an intrinsic gluon. The effective physical scale for this process is the scale of the Pomeron which couples to the constituent gluon over a distance $\sim 1 / \sqrt{\alpha_{P}^{\prime}}$, therefore described by the wave function $\Phi_{\tau}^{g}(z) \sim z^{\tau} e^{-\lambda_{g} z^{2} / 2}$ [21]. Upon substituting in (11) the $x$-integral representation of the bulk-to-boundary propagator (9) we find

$$
A_{\tau}^{g}\left(Q^{2}\right)=\frac{1}{N_{\tau}} B\left(\tau-1,2-\alpha_{P}\left(Q^{2}\right)\right),
$$

with $N_{\tau}=B\left(\tau-1,2-\alpha_{P}(0)\right)$, the result of the generalized Veneziano model $[26,29,30]$ for a spin-two current.

In writing (12) we have assumed that only the dilaton profile describing Pomeron exchange sets the scale when 
computing the gluon GFFs and GPDs, while only the dilaton corresponding to Reggeon exchange needs to be considered when finding the EM FFs and quark GPDs [27]. In (12) we have also shifted the Pomeron intercept to its physical value $\alpha_{P}(0) \approx 1$, since the expression which follows from the holographic result (11) leads to a zero intercept. This procedure, which has been successfully applied to our treatment of the EM FF [27], maintains the analytic properties of the GFF: It amounts to a displacement of the timelike poles of the exchanged particles in the $t$-channel $[27,66]$. In fact, for integer twist the GFF (12) is expressed as a product of $\tau-1$ timelike poles located at

$$
-Q^{2}=M_{n}^{2}=\frac{1}{\alpha_{P}^{\prime}}\left(n+2-\alpha_{P}(0)\right)
$$

the radial excitation spectrum of the spin-two Pomeron. The lowest state in this trajectory, the $2^{++}$, has the mass $M \simeq 1.92 \mathrm{GeV}$, compared with the lattice results $M \simeq$ (2.15-2.4) GeV [40,67-69].

The predictions for the GFF, $A^{g}\left(Q^{2}\right)$, for the nucleon and pion are presented in Fig. 1. We find for the gluon mass squared radius

$$
\left\langle r_{g}^{2}\right\rangle=\left.\frac{6}{A^{g}(0)} \frac{d A^{g}(t)}{d t}\right|_{t=0}
$$

$\left\langle r_{g}^{2}\right\rangle_{p}=2.93 / \lambda_{g}=(0.34 \mathrm{fm})^{2} \quad$ and $\quad\left\langle r_{g}^{2}\right\rangle_{\pi}=2.41 / \lambda_{g}=$ $(0.31 \mathrm{fm})^{2}$ for the proton and pion, indicating a gluonmass distribution concentrated in a rather small region

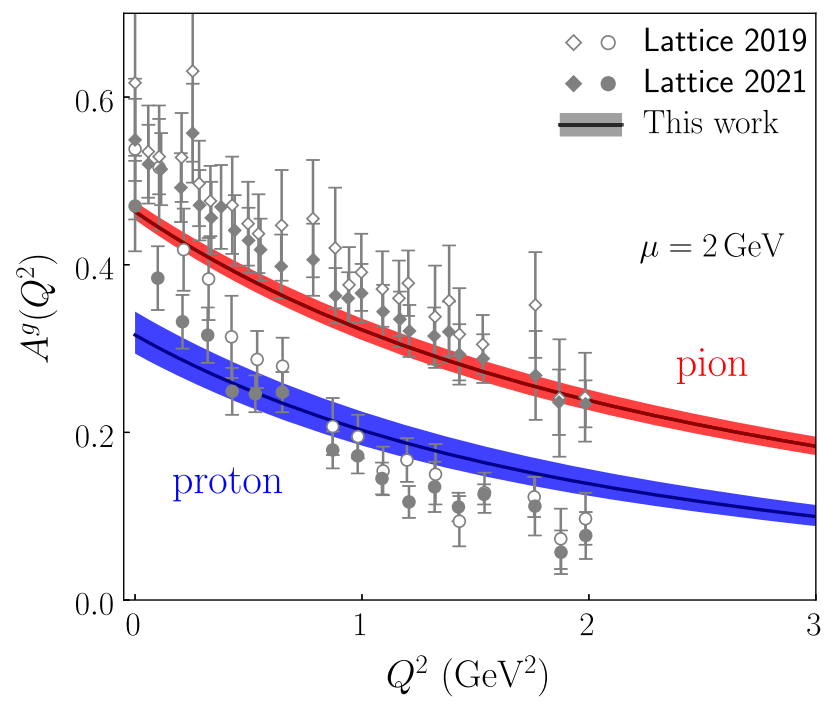

FIG. 1. Gluon gravitational form factor $A^{g}\left(Q^{2}\right)$ of the proton (blue) and the pion (red) in comparison with lattice QCD calculations in Refs. [70,71]. The value $A^{g}(0)$ corresponds to the momentum fraction carried by gluons: $0.316 \pm 0.025$ for the proton and $0.463 \pm 0.011$ for the pion at $\mu=2 \mathrm{GeV}$. The bands indicate the uncertainty from the variation of $\lambda_{g}$ by $\pm 5 \%$ and the normalization from the momentum sum rule. compared with the spread of the charge [40], and also smaller than the proton mass radius found in [63,72-74]. The normalization used in Fig. 1 is discussed below.

\section{GLUON DISTRIBUTION FUNCTIONS}

Recent calculations of the gluon distribution functions in the hadrons have been performed using holographic approaches, such as in [75-78]. Following the unified approach advanced in $[27,34]$ we determine in the present work the unpolarized gluon distributions in the nucleon and pion without introducing any new free parameters. In the nonperturbative domain, low virtuality gluons interact strongly with each other to generate the color confinement potential and one cannot distinguish individual gluon quanta. At higher virtualities constituent gluons appear as new degrees of freedom. The lowest gluonic Fock state of the proton is $|u u d g\rangle$ and, for simplicity, we consider this Fock state to be the dominant contribution to the intrinsic gluon distribution.

Using (12) and the integral representation of the Beta function, the gluon GFF $A_{\tau}(t)$ can be written in the reparametrization invariant form

$$
A_{\tau}^{g}(t)=\frac{1}{N_{\tau}} \int_{0}^{1} d x w^{\prime}(x) w(x)^{1-\alpha_{P}(t)}[1-w(x)]^{\tau-2},
$$

provided that $w(x)$ satisfies the constraints $w(0)=0$, $w(1)=1$ and $w^{\prime}(x) \geq 0[27,34]$. The GFF can also be expressed as the first moment of the gluon GPD at zero skewness, $H_{\tau}^{g}(x, t) \equiv H_{\tau}^{g}(x, \xi=0, t)$,

$$
\begin{aligned}
A_{\tau}^{g}(t) & =\int_{0}^{1} x d x H_{\tau}^{g}(x, t) \\
& =\int_{0}^{1} x d x g_{\tau}(x) e^{t f(x)},
\end{aligned}
$$

where $f(x)$ is the profile function and $g_{\tau}(x)$ is the collinear gluon PDF of twist- $\tau$. Comparing (16) with the holographic expression (15) we find that both functions, $f(x)$ and $g_{\tau}(x)$, are determined in terms of the reparametrization function, $w(x)$, by

$$
\begin{gathered}
f_{P}(x)=\alpha_{P}^{\prime} \log \left(\frac{1}{w(x)}\right) \\
g_{\tau}(x)=\frac{1}{N_{\tau}} \frac{w^{\prime}(x)}{x}[1-w(x)]^{\tau-2} w(x)^{1-\alpha_{P}(0)}
\end{gathered}
$$

where $g_{\tau}(x)$ is normalized by $\int_{0}^{1} d x x g_{\tau}(x)=1$.

If we identify $x$ with the gluon LF momentum fraction, physical constraints on $w(x)$ are imposed at small and large- $x$ [79]. At $x \rightarrow 0, w(x) \sim x$ from Regge theory [80], and at $x \rightarrow 1$ from the inclusive-exclusive counting rule [33], $g_{\tau}(x) \sim(1-x)^{2 \tau-3}$, which fixes $w^{\prime}(1)=0$. 
The leading $(1-x)$-exponent determined in [81] by fitting the NNPDF gluon distribution [82] is consistent with the large- $x$ counting rule.

The gluon distribution of the proton can be expressed as the sum of contributions from all Fock states, $g(x)=\sum_{\tau} c_{\tau} g_{\tau}(x)$, where the coefficients, $c_{\tau}$, represent the normalization of each Fock component. In practice, one has to apply a truncation up to some value of $\tau$. In this study we only keep the leading term, $\tau=4$, and determine the coefficient $c_{\tau=4}$ using the momentum sum rule

$$
\int_{0}^{1} d x x\left[g(x)+\sum_{q} q(x)\right]=1,
$$

where $q$ runs over all quark flavors. It also corresponds to the sum rule of the helicity-conserving GFF $A(t)$,

$$
A^{g}(0)+\sum_{q} A^{q}(0)=1,
$$

which is a measure of the momentum fraction carried by each constituent. Similarly the helicity-flip GFF $B(t)$ provides a measure of the orbital angular momentum carried by each constituent of a hadron at $t=0$. The constraint $B^{g}(0)+\sum_{q} B^{q}(0)=0$ was originally derived from the equivalence principle [83] and can be formally derived Fock state by Fock state in LF quantization [84].

Taking the quark distributions and the reparametrization function $w(x)$ from Ref. [34], $w(x)=x^{1-x} \exp \left[-b(1-x)^{2}\right]$ with $b=0.48 \pm 0.04$, we determine $c_{\tau=4}=0.225 \pm 0.014$ and thus the gluon distribution at the input scale $\mu_{0}=1.06 \pm 0.15 \mathrm{GeV}$. Together with the quark distributions, we evolve the gluon distribution to $\mu^{2}=10 \mathrm{GeV}^{2}$. The model results are compared in Fig. 2 with global analyses of gluon PDFs $[82,85,86]$. Contributions from higher Fock states are expected to be suppressed at large $x$ and may affect the overall normalization through the momentum sum rule, thus suppressing the gluon distribution at large $x$ while enhancing the distribution at small $x$. Our nonperturbative results falloff as $(1-x)^{5}$ at large $x$ from the leading twist-four term in (19). In contrast, the perturbative QCD results incorporate a $(1-x)^{4}$ falloff from hard gluon transfer to the spectator quarks [87].

Incorporating the universality of our approach, we now compute the gluon distribution in the pion. Similar to the case of the proton, we only consider the lowest $\tau=3$ Fock state $|u \bar{d} g\rangle$ with one constituent gluon. The coupling of the Pomeron to the hadrons depends on the vertex, but the trajectory $\alpha_{P}(t)(1)$ is the same and unique to the Pomeron. Considering the valence quark distributions determined in Ref. [27],

$$
|\pi\rangle=\sqrt{0.875}|u \bar{d}\rangle+\sqrt{0.125}|u \bar{d} q \bar{q}\rangle,
$$

we express the total quark distribution as
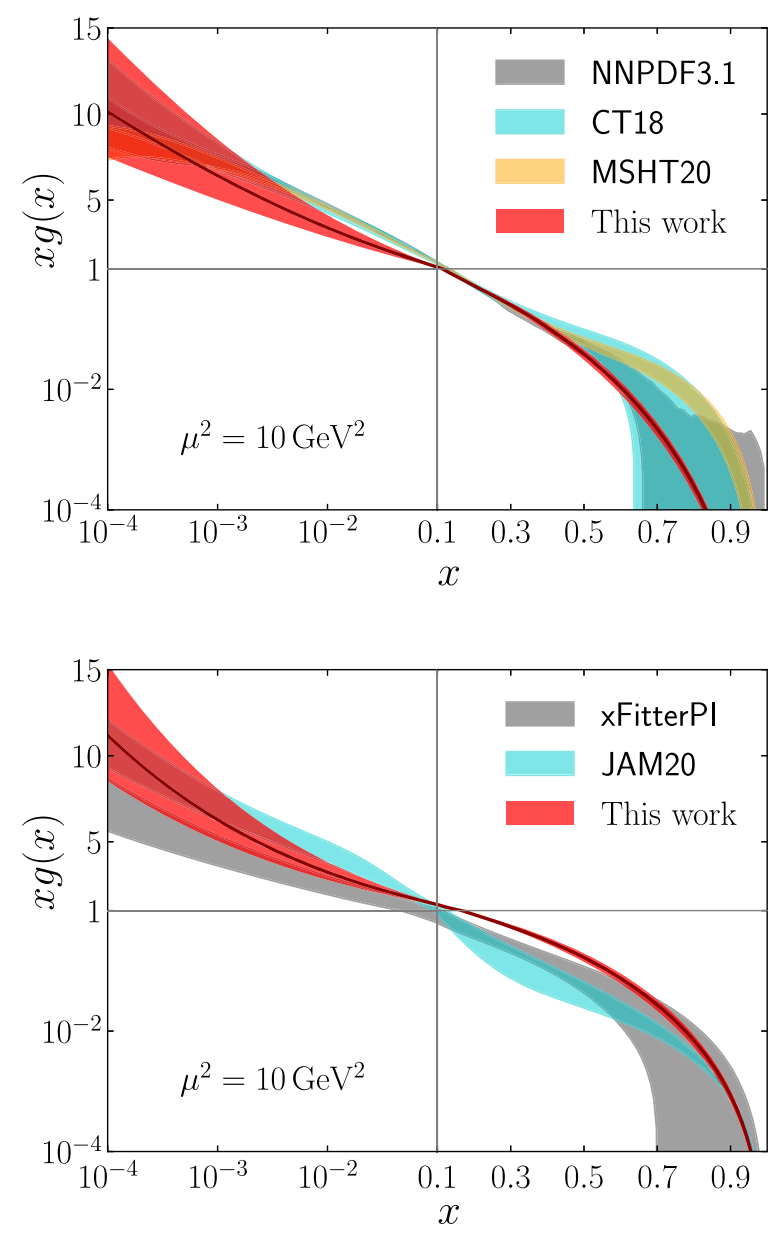

FIG. 2. Comparison of the gluon distribution in the proton (up) and pion (down) with global fits. The red bands indicate the theoretical uncertainty from the initial scale $\mu_{0}$ and the gluon coefficient $c_{\tau}$ from the momentum sum rule.

$$
\begin{aligned}
q_{\pi}(x) & \equiv q_{u}(x)+q_{d}(x)+q_{\bar{u}}(x)+q_{\bar{d}}(x) \\
& \simeq 2 \times 0.875 q_{\tau=2}(x)+4 \times 0.125 q_{\tau=4}(x) \\
& =1.75 q_{\tau=2}(x)+0.5 q_{\tau=4}(x) .
\end{aligned}
$$

Using the momentum sum rule we obtain the gluon distribution of the pion as $g_{\pi}(x)=(0.429 \pm 0.007) g_{\tau=3}(x)$. We show in Fig. 2 the results evolved to $\mu^{2}=10 \mathrm{GeV}^{2}$ and a comparison with global analyses $[88,89]$. We note that the overall normalization of the gluon distribution from our calculation seems overestimated in comparison with some recent global analyses, which may arise from neglecting higher Fock states for the gluon GFF.

\section{CONCLUSION AND OUTLOOK}

The light-front holographic extension presented in this article allows us to give a simultaneous description of the intrinsic gluon distributions, GPD and PDF, and the gluon GFF $A_{g}(t)$, within a unified nonperturbative framework, for both the nucleon and pion. The actual computations are 
based on the holographic coupling of the spin-two soft Pomeron to the hadron energy-momentum tensor, constrained by the Veneziano structure. The comparison of our theoretical predictions, after DGLAP evolution, with global analyses and recent lattice computations clearly demonstrates the predictive power of this new framework.

In this article we have only used the parameters of the soft Pomeron. For hard processes the contribution of a hard Pomeron with a much larger intercept and smaller slope also seems to be necessary [43]. However, after evolution, our results describe the full gluon distribution in accordance with phenomenological determinations, see Fig. 2. This supports attempts to use the gauge/gravity duality $[18,55,90]$ to describe hard and soft diffractive processes within a unified framework. The properties of the Pomeron depend then on the kinematic regime of the scattering process [55,90,91].

The gluonic and quark distributions of hadrons are shown to have significantly different effective QCD scales and sizes compared to their electromagnetic distributions. The twist-three Fock state $|q \bar{q} g\rangle$ in the pion $(|q q q g\rangle$ for the nucleon) is responsible for the intrinsic gluon distribution at the initial evolution scale where the Pomeron probe couples strongly with the constituent gluon. However, this Fock component does not contribute significantly to the quark
GPD since the EM probe does not resolve the deeply bound constituent gluon [92], and thus it is effectively included in the twist-two $q \bar{q}$ state ( $q q q$ for the nucleon). Our results for $A(t)$ can be extended to the other two invariant GFFs $B(t)$ and $C(t)$ [3]. Of particular relevance is the coupling of the scalar Pomeron trajectory-with similar slope, but different intercept, to determine the form factor $C(t)[74,75]$. It can allow us to gain further insights into the distribution of internal shear forces and pressure inside the proton and therefore of its dynamical stability.

\section{ACKNOWLEDGMENTS}

R. S. S. thanks Patrick Barry for providing the JAM20 gluon distribution of the pion. T. L. is supported in part by the National Natural Science Foundation of China under Contract No. 12175117. R. S. S. is supported by U.S. DOE Grant No. DE-FG02-04ER41302 and in part by the U.S. Department of Energy Contract No. DE-AC0506OR23177, under which Jefferson Science Associates, LLC, manages and operates Jefferson Lab. S. J. B. is supported in part by the Department of Energy Contract No. DE-AC02-76SF00515.
[1] I. Y. Kobzarev and L. B. Okun, Gravitational interaction of fermions, Zh. Eksp. Teor. Fiz. 43, 1904 (1962).

[2] S. Weinberg, Photons and gravitons in $S$-matrix theory: Derivation of charge conservation and equality of gravitational and inertial mass, Phys. Rev. 135, B1049 (1964).

[3] H. Pagels, Energy-momentum structure form factors of particles, Phys. Rev. 144, 1250 (1966).

[4] D. Müller, D. Robaschik, B. Geyer, F. M. Dittes, and J. Hořejši, Wave functions, evolution equations and evolution kernels from light-ray operators of QCD, Fortschr. Phys. 42, 101 (1994).

[5] X. D. Ji, Gauge-Invariant Decomposition of Nucleon Spin, Phys. Rev. Lett. 78, 610 (1997).

[6] A. V. Radyushkin, Nonforward parton distributions, Phys. Rev. D 56, 5524 (1997).

[7] M. V. Polyakov, Generalized parton distributions and strong forces inside nucleons and nuclei, Phys. Lett. B 555, 57 (2003).

[8] M. V. Polyakov and P. Schweitzer, Forces inside hadrons: Pressure, surface tension, mechanical radius, and all that, Int. J. Mod. Phys. A 33, 1830025 (2018).

[9] V. D. Burkert, L. Elouadrhiri, and F. X. Girod, The pressure distribution inside the proton, Nature (London) 557, 396 (2018).

[10] H. M. Georgi, S. L. Glashow, M. E. Machacek, and D. V. Nanopoulos, Higgs Bosons from Two-Gluon Annihilation in Proton-Proton Collisions, Phys. Rev. Lett. 40, 692 (1978).
[11] S. J. Brodsky, E. Chudakov, P. Hoyer, and J. M. Laget, Photoproduction of charm near threshold, Phys. Lett. B 498, 23 (2001).

[12] S. J. Brodsky, I. A. Schmidt, and G. F. de Téramond, Nuclear-Bound Quarkonium, Phys. Rev. Lett. 64, 1011 (1990).

[13] D. Kharzeev, H. Satz, A. Syamtomov, and G. Zinovjev, J/ $\psi$ photoproduction and the gluon structure of the nucleon, Eur. Phys. J. C 9, 459 (1999).

[14] G. F. de Téramond and S. J. Brodsky, Longitudinal dynamics and chiral symmetry breaking in holographic light-front QCD, arXiv:2103.10950.

[15] A. C. Aguilar, Z. Ahmed, C. Aidala, S. Ali, V. Andrieux, J. Arrington, A. Bashir, V. Berdnikov, D. Binosi, L. Chang et al., Pion and kaon structure at the electron-ion collider, Eur. Phys. J. A 55, 190 (2019).

[16] A. Accardi et al., Electron ion collider: The next QCD frontier, Eur. Phys. J. A 52, 268 (2016).

[17] R. Abdul Khalek et al., Science requirements and detector concepts for the electron-ion collider: EIC yellow report, arXiv:2103.05419.

[18] J. M. Maldacena, The large- $N$ limit of superconformal field theories and supergravity, Int. J. Theor. Phys. 38, 1113 (1999); Adv. Theor. Math. Phys. 2, 231 (1998).

[19] S. J. Brodsky and G. F. de Téramond, Hadronic Spectra and Light-Front Wave Functions in Holographic QCD, Phys. Rev. Lett. 96, 201601 (2006). 
[20] G. F. de Téramond and S. J. Brodsky, Light-Front Holography: A First Approximation to QCD, Phys. Rev. Lett. 102, 081601 (2009).

[21] S. J. Brodsky, G. F. de Téramond, H. G. Dosch, and J. Erlich, Light-front holographic QCD and emerging confinement, Phys. Rep. 584, 1 (2015).

[22] S. Fubini and E. Rabinovici, Superconformal quantum mechanics, Nucl. Phys. B245, 17 (1984).

[23] G. F. de Téramond, H. G. Dosch, and S. J. Brodsky, Baryon spectrum from superconformal quantum mechanics and its light-front holographic embedding, Phys. Rev. D 91, 045040 (2015).

[24] H. G. Dosch, G. F. de Téramond, and S. J. Brodsky, Superconformal baryon-meson symmetry and light-front holographic QCD, Phys. Rev. D 91, 085016 (2015).

[25] S. J. Brodsky, G. F. de Téramond, and H. G. Dosch, Lightfront holography and supersymmetric conformal algebra: A novel approach to hadron spectroscopy, structure, and dynamics, arXiv:2004.07756.

[26] G. Veneziano, Construction of a crossing-symmetric, and Regge-behaved amplitude for linearly rising trajectories, Nuovo Cimento A 57, 190 (1968).

[27] G. F. de Téramond, T. Liu, R. S. Sufian, H. G. Dosch, S. J. Brodsky, and A. Deur, Universality of Generalized Parton Distributions in Light-Front Holographic QCD, Phys. Rev. Lett. 120, 182001 (2018).

[28] L. Zou and H. G. Dosch, A very practical guide to light-front holographic QCD, arXiv:1801.00607.

[29] M. Ademollo and E. Del Giudice, Nonstrong amplitudes in a Veneziano-type model, Nuovo Cimento A 63, 639 (1969).

[30] P. V. Landshoff and J. C. Polkinghorne, The scaling law for deep inelastic scattering in a new Veneziano-like amplitude, Nucl. Phys. B19, 432 (1970).

[31] S. J. Brodsky and G. R. Farrar, Scaling Laws at Large Transverse Momentum, Phys. Rev. Lett. 31, 1153 (1973).

[32] V. A. Matveev, R. M. Muradian, and A. N. Tavkhelidze, Automodellism in the large-angle elastic scattering and structure of hadrons, Lett. Nuovo Cimento 7, 719 (1973).

[33] S. D. Drell and T. M. Yan, Connection of Elastic Electromagnetic Nucleon Form-Factors at Large $Q^{2}$ and Deep Inelastic Structure Functions Near Threshold, Phys. Rev. Lett. 24, 181 (1970).

[34] T. Liu, R. S. Sufian, G. F. de Téramond, H. G. Dosch, S. J. Brodsky, and A. Deur, Unified Description of Polarized and Unpolarized Quark Distributions in the Proton, Phys. Rev. Lett. 124, 082003 (2020).

[35] R. S. Sufian, T. Liu, G. F. de Téramond, H. G. Dosch, S. J. Brodsky, A. Deur, M. T. Islam, and B.-Q. Ma, Nonperturbative strange-quark sea from lattice QCD, light-front holography, and meson-baryon fluctuation models, Phys. Rev. D 98, 114004 (2018).

[36] R. S. Sufian, T. Liu, A. Alexandru, S. J. Brodsky, G. F. de Téramond, H. G. Dosch, T. Draper, K.-F. Liu, and Y.-B. Yang, Constraints on charm-anticharm asymmetry in the nucleon from lattice QCD, Phys. Lett. B 808, 135633 (2020).

[37] J. Rojo, Progress in the NNPDF global analyses of proton structure, arXiv:2104.09174.

[38] A. Di Giacomo, H. G. Dosch, V. I. Shevchenko, and Y. A. Simonov, Field correlators in QCD: Theory and applications, Phys. Rep. 372, 319 (2002).
[39] G. S. Bali, Casimir scaling of SU(3) static potentials, Phys. Rev. D 62, 114503 (2000).

[40] P. A. Zyla et al. (Particle Data Group), Review of particle physics, Prog. Theor. Exp. Phys. 2020, 083C01 (2020).

[41] L. V. Gribov, E. M. Levin, and M. G. Ryskin, Semihard processes in QCD, Phys. Rep. 100, 1 (1983).

[42] B. Badelek, M. Krawczyk, K. Charchula, and J. Kwiecinski, Small- $x$ physics in deep-inelastic lepton-hadron scattering, Rev. Mod. Phys. 64, 927 (1992).

[43] S. Donnachie, H. G. Dosch, O. Nachtmann, and P. Landshoff, Pomeron Physics and QCD, in Pomeron Physics and QCD, in Cambridge Monographs on Particle Physics, Nuclear Physics and Cosmology series Vol. 19 (Cambridge University Press, Cambridge, England, 2002).

[44] L. Adamczyk et al. (STAR Collaboration), Single spin asymmetry $A_{N}$ in polarized proton-proton elastic scattering at $\sqrt{s}=200 \mathrm{GeV}$, Phys. Lett. B 719, 62 (2013).

[45] C. Ewerz, M. Maniatis, and O. Nachtmann, A model for soft high-energy scattering: Tensor pomeron and vector odderon, Ann. Phys. (Amsterdam) 342, 31 (2014).

[46] C. Ewerz, P. Lebiedowicz, O. Nachtmann, and A. Szczurek, Helicity in proton-proton elastic scattering and the spin structure of the Pomeron, Phys. Lett. B 763, 382 (2016).

[47] P. Lebiedowicz, O. Nachtmann, and A. Szczurek, Exclusive diffractive production of $\pi^{+} \pi^{-} \pi^{+} \pi^{-}$via the intermediate $\sigma \sigma$ and $\rho \rho$ states in proton-proton collisions within tensor Pomeron approach, Phys. Rev. D 94, 034017 (2016).

[48] D. Britzger, C. Ewerz, S. Glazov, O. Nachtmann, and S. Schmitt, The tensor Pomeron and low- $x$ deep inelastic scattering, Phys. Rev. D 100, 114007 (2019).

[49] A. Donnachie and P. V. Landshoff, Total cross-sections, Phys. Lett. B 296, 227 (1992).

[50] E. A. Kuraev, L. N. Lipatov, and V. S. Fadin, The Pomeranchuk singularity in nonabelian gauge theories, Sov. Phys. JETP 45, 199 (1977).

[51] I. I. Balitsky and L. N. Lipatov, The Pomeranchuk singularity in quantum chromodynamics, Sov. J. Nucl. Phys. 28, 822 (1978).

[52] R. Kirschner and L. N. Lipatov, Double logarithmic asymptotics and Regge singularities of quark amplitudes with flavour exchange, Nucl. Phys. B213, 122 (1983).

[53] A. H. Mueller and J.W. Qiu, Gluon recombination and shadowing at small values of $x$, Nucl. Phys. B268, 427 (1986).

[54] Y. V. Kovchegov, Small- $x F_{2}$ structure function of a nucleus including multiple pomeron exchanges, Phys. Rev. D 60, 034008 (1999).

[55] R. C. Brower, J. Polchinski, M. J. Strassler, and C. I. Tan, The Pomeron and gauge/string duality, J. High Energy Phys. 12 (2007) 005.

[56] L. Cornalba and M. S. Costa, Saturation in deep inelastic scattering from AdS/CFT, Phys. Rev. D 78, 096010 (2008).

[57] S. K. Domokos, J. A. Harvey, and N. Mann, The Pomeron contribution to $p p$ and $p \bar{p}$ scattering in AdS/QCD, Phys. Rev. D 80, 126015 (2009).

[58] R. C. Brower, M. Djuric, I. Sarcevic, and C. I. Tan, Stringgauge dual description of deep inelastic scattering at small$x$, J. High Energy Phys. 11 (2010) 051.

[59] M. S. Costa and M. Djuric, Deeply virtual Compton scattering from gauge/gravity duality, Phys. Rev. D 86, 016009 (2012). 
[60] M. S. Costa, M. Djurić, and N. Evans, Vector meson production at low $x$ from gauge/gravity duality, J. High Energy Phys. 09 (2013) 084.

[61] A. Amorim, M. S. Costa, and M. Järvinen, Regge theory in a holographic dual of QCD in the Veneziano limit, J. High Energy Phys. 07 (2021) 065.

[62] There exist many computations of glueballs in top-down holographic models, see for example, C. Csaki, H. Ooguri, Y. Oz, and J. Terning, Glueball mass spectrum from supergravity, J. High Energy Phys. 01 (1999) 017; and also in bottom-up models starting from H. Boschi-Filho, N. R. F. Braga, and H. L. Carrion, Glueball Regge trajectories from gauge-string duality and the Pomeron, Phys. Rev. D 73, 047901 (2006); For a recent computation, see for example, M. Rinaldi and V. Vento, Meson and glueball spectroscopy within the graviton soft wall model, Phys. Rev. D 104, 034016 (2021), and references therein.

[63] Z. Abidin and C. E. Carlson, Gravitational form factors of vector mesons in an AdS/QCD model, Phys. Rev. D 77, 095007 (2008); Nucleon electromagnetic and gravitational form factors from holography, Phys. Rev. D 79, 115003 (2009).

[64] S. J. Brodsky and G. F. de Téramond, Light-front dynamics and AdS/QCD correspondence: Gravitational form factors of composite hadrons, Phys. Rev. D 78, 025032 (2008).

[65] A. Karch, E. Katz, D. T. Son, and M. A. Stephanov, Linear confinement and AdS/QCD, Phys. Rev. D 74, 015005 (2006).

[66] See also, S. S. Afonin and T. D. Solomko, Theory of bottom-up holographic models for linear Regge trajectories, arXiv:2106 .01846.

[67] C. J. Morningstar and M. J. Peardon, Glueball spectrum from an anisotropic lattice study, Phys. Rev. D 60, 034509 (1999).

[68] H. B. Meyer and M. J. Teper, Glueball Regge trajectories and the Pomeron: A lattice study, Phys. Lett. B 605, 344 (2005).

[69] H. B. Meyer, Glueball Regge trajectories, Ph. D. Thesis, University of Oxford, 2004 [arXiv:hep-lat/0508002].

[70] P. E. Shanahan and W. Detmold, Gluon gravitational form factors of the nucleon and the pion from lattice QCD, Phys. Rev. D 99, 014511 (2019).

[71] D. A. Pefkou, D. C. Hackett, and P. E. Shanahan, Gluon gravitational structure of hadrons of different spin, arXiv: 2107.10368. The 2021 LQCD results were uploaded to arXiv after the submission of our article, but are included here together with the 2020 LQCD data in [70] for a meaningful comparison with our results.

[72] Y. Guo, X. Ji, and Y. Liu, QCD analysis of near-threshold photon-proton production of heavy quarkonium, Phys. Rev. D 103, 096010 (2021).

[73] D. E. Kharzeev, Mass radius of the proton, Phys. Rev. D 104, 054015 (2021).

[74] K. A. Mamo and I. Zahed, Nucleon mass radii and distribution: Holographic QCD, Lattice QCD and GlueX data, Phys. Rev. D 103, 094010 (2021).

[75] K. A. Mamo and I. Zahed, Diffractive photoproduction of $J / \psi$ and $\Upsilon$ using holographic QCD: Gravitational form factors and GPD of gluons in the proton, Phys. Rev. D 101, 086003 (2020).

[76] A. Watanabe, T. Sawada, and M. Huang, Extraction of gluon distributions from structure functions at small $x$ in holographic QCD, Phys. Lett. B 805, 135470 (2020).

[77] V. E. Lyubovitskij and I. Schmidt, Gluon parton densities in soft-wall AdS/QCD, Phys. Rev. D 103, 094017 (2021).

[78] J. Lan, K. Fu, C. Mondal, X. Zhao, and J. P. Vary, Light mesons with one dynamical gluon on the light front, arXiv: 2106.04954.

[79] R. D. Ball, E. R. Nocera, and J. Rojo, The asymptotic behaviour of parton distributions at small and large $x$, Eur. Phys. J. C 76, 383 (2016).

[80] T. Regge, Introduction to complex orbital momenta, Nuovo Cimento 14, 951 (1959).

[81] R. S. Sufian, T. Liu, and A. Paul, Gluon distributions and their applications to Ioffe-time distributions, Phys. Rev. D 103, 036007 (2021).

[82] R. D. Ball et al. (NNPDF Collaboration), Parton distributions from high-precision collider data, Eur. Phys. J. C 77, 663 (2017).

[83] O. V. Teryaev, Spin structure of nucleon and equivalence principle, arXiv:hep-ph/9904376.

[84] S. J. Brodsky, D. S. Hwang, B.-Q. Ma, and I. Schmidt, Light-cone representation of the spin and orbital angular momentum of relativistic composite systems, Nucl. Phys. B593, 311 (2001).

[85] T. J. Hou, J. Gao, T. J. Hobbs, K. Xie, S. Dulat, M. Guzzi, J. Huston, P. Nadolsky, J. Pumplin, C. Schmidt et al., New CTEQ global analysis of quantum chromodynamics with high-precision data from the LHC, Phys. Rev. D 103, 014013 (2021).

[86] S. Bailey, T. Cridge, L. A. Harland-Lang, A. D. Martin, and R.S. Thorne, Parton distributions from LHC, HERA, Tevatron and fixed target data: MSHT20 PDFs, Eur. Phys. J. C 81, 341 (2021).

[87] S. J. Brodsky, M. Burkardt, and I. Schmidt, Perturbative QCD constraints on the shape of polarized quark and gluon distributions, Nucl. Phys. B441, 197 (1995).

[88] I. Novikov, H. Abdolmaleki, D. Britzger, A. Cooper-Sarkar, F. Giuli, A. Glazov, A. Kusina, A. Luszczak, F. Olness, P. Starovoitov et al., Parton distribution functions of the charged pion within the xFitter framework, Phys. Rev. D 102, 014040 (2020).

[89] N. Y. Cao, P. C. Barry, N. Sato, and W. Melnitchouk (JAM Collaboration), Towards the three-dimensional parton structure of the pion: Integrating transverse momentum data into global QCD analysis, Phys. Rev. D 103, 114014 (2021).

[90] Y. Hatta, E. Iancu, and A. H. Mueller, Deep inelastic scattering at strong coupling from gauge/string duality: The saturation line, J. High Energy Phys. 01 (2008) 026.

[91] H. G. Dosch and E. Ferreira, Diffractive electromagnetic processes from a Regge point of view, Phys. Rev. D 92, 034002 (2015).

[92] S. J. Brodsky and G. F. de Téramond, AdS/QCD, light-front holography, and sublimated gluons, Proc. Sci. QCD-TNTII2011 (2011) 008 [arXiv:1112.4212]. 